\title{
Final Technical Report \\ For Closeout of Award No. DE-FG02-03ER41250
}

TITLE OF RESEARCH: Advanced Beam-Dynamics Simulation Tools for RIA Research and Development for the Rare Isotope Accelerator

NAME OF INSTITUTION: Michigan State University

PRINCIPAL INVESTIGATOR:

Richard C. York

National Superconducting Cyclotron Laboratory

Michigan State University

East Lansing, MI 48824-1321

Phone/Fax: 517-333-6325/ 517-353-5967

Email: york@nscl.msu.edu

Michigan State University (MSU) activities in support of this grant were made as part of a larger collaboration including Los Alamos National Laboratory, Argonne National Laboratory, and Lawrence Berkeley National Laboratory.

The main task of the collaboration was the development of an end-to-end multiparticle beam-dynamics simulation tool for computation of beam losses in the RIA driver linac. As the first part of this task, it was planned to modify PARMTEQ for multicharge-state beam-dynamics simulation in the LEBT and RFQ. The next part of this task was to develop a new multiparticle parallel code to model the superconducting driver linac. The output particle distributions from PARMTEQ could then be used as input for simulations through the superconducting linac, using the new parallel code with different random number seeds.

The stripping of heavy ions is proposed for the RIA driver linac to increase acceleration efficiency. MSU developed a complete charge stripping foil model to evaluate the impact of the stripping foil on the beam transverse and longitudinal emittance. The stripping foil model was developed in LANA [1] and included the effects of elastic and inelastic scattering, ionization energy loss, and thickness variation in the stripping foil using the code SRIM [2]. This model was provided to the collaboration for inclusion in the new simulation tools.

Benchmarking information in support of the new code development was provided by supplying the collaboration with the MSU driver linac model consisting of input information for LANA and DIMAD [3.] The output of simulations using LANA and DIMAD were also provided. As an element of these activities, LANA was modified to provide simulation results with high statistics. The simulation results from the newly developed simulation tools and those of MSU gave statistically equivalent results. 
Finally, a version of the newly developed IMPACT 3D simulation package was installed and commissioned at MSU to provide another channel for simulation comparison and critical input to the new code development.

\section{REFERENCES}

[1.] D.V.Gorelov and P.N.Ostroumov, "Applications of the LANA Code for Design of Ion Linacs", Proc. of the EPAC"96 Conf., Sitges, Spain, (1996) p.1271.

\section{[2.] SRIM:http://www.srim.org}

[3.] User Guide to the Program DIMAD, SLAC Report 285, UC-28 (1985). 